\title{
A hemispherical power asymmetry from inflation
}

\author{
Adrienne L. Erickcek, Marc Kamionkowski, and Sean M. Carroll \\ California Institute of Technology, Mail Code 130-33, Pasadena, California 91125, USA \\ (Received 3 June 2008; revised manuscript received 10 July 2008; published 16 December 2008)
}

\begin{abstract}
Measurements of cosmic microwave background temperature fluctuations by the Wilkinson Microwave Anisotropy Probe indicate that the fluctuation amplitude in one half of the sky differs from the amplitude in the other half. We show that such an asymmetry cannot be generated during single-field slow-roll inflation without violating constraints to the homogeneity of the Universe. In contrast, a multifield inflationary theory, the curvaton model, can produce this power asymmetry without violating the homogeneity constraint. The mechanism requires the introduction of a large-amplitude superhorizon perturbation to the curvaton field, possibly a preinflationary remnant or a superhorizon curvaton-web structure. The model makes several predictions, including non-Gaussianity and modifications to the inflationary consistency relation, that will be tested with forthcoming cosmic microwave background experiments.
\end{abstract}

DOI: 10.1103/PhysRevD.78.123520

PACS numbers: $98.80 . \mathrm{Cq}, 98.70 . \mathrm{Vc}$

\section{INTRODUCTION}

Inflation provides a compelling description of the early Universe [1]. The temperature fluctuations in the cosmic microwave background (CMB) [2,3] and the distribution of galaxies [4] agree well with inflationary predictions. However, there is an anomaly in the CMB: measurements from the Wilkinson Microwave Anisotropy Probe (WMAP) [3] indicate that the temperature-fluctuation amplitude is larger, by roughly $10 \%$, in one hemisphere than in the other [5]. Fewer than $1 \%$ of simulated isotropic fluctuation maps exhibit such an asymmetry, and the asymmetry cannot be attributed to any known astrophysical foreground or experimental artifact. As opposed to the "axis of evil" [6], an apparent alignment of only the lowest multipole moments, this asymmetry has gone largely unnoticed (although see $[7,8]$ ), and it warrants further theoretical consideration.

In the standard inflation scenario, the Universe undergoes a very long inflationary expansion before the comoving observable Universe exits the horizon during inflation. Thus, any remnants of a preinflationary Universe were inflated away before there could be observable consequences. This accounts for the smoothness of the primordial Universe as well as its flatness. It also suggests that primordial density perturbations should show no preferred direction. The existence of a hemispherical power asymmetry in the $\mathrm{CMB}$ challenges this basic prediction of inflation.

A superhorizon perturbation would introduce a preferred direction in the Universe and has been considered as a possible origin of the axis of evil [9]. In this article, we investigate how the hemispherical power asymmetry could result from a superhorizon perturbation during inflation, as depicted in Fig. 1. Since the amplitude of the primordial fluctuations depends on the background value of the fluctuating inflationary field, a large-amplitude superhorizon fluctuation would generate a power asymmetry by varying the background value of the field across the observable Universe. Of course, the superhorizon fluctuation would make the Universe inhomogeneous, and the near uniformity of the CMB constrains such departures from homogeneity [10].

We begin by showing in Sec. II that the power asymmetry cannot be reconciled with single-field slow-roll inflation without violating constraints to the homogeneity of the Universe. We then consider an alternative inflationary theory, the curvaton model [11], which has been suggested as a possible source of a power asymmetry [7]. In Sec. III, we demonstrate that a superhorizon fluctuation in the curvaton field can generate the observed asymmetry without violating the homogeneity constraints. The required superhorizon fluctuation in the curvaton field may occur, for example, as a remnant of the preinflationary epoch or as a signature of superhorizon curvaton-web structures [12]. The proposed model predicts several signatures, which may soon be tested, in the CMB. We discuss these signatures and summarize our findings in Sec. IV.

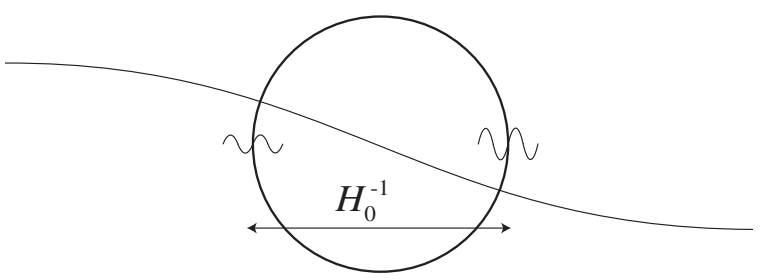

FIG. 1. Measurements of temperature fluctuations in the CMB show that the rms temperature-fluctuation amplitude is larger in one side of the sky than in the other. We investigate here whether this may arise as a consequence of a large-scale mode of an inflaton or a curvaton. 


\section{SINGLE-FIELD MODELS}

Inflation postulates that the energy density in the early Universe was dominated by a scalar field $\phi$, the inflaton. The energy density is due to kinetic energy $(1 / 2) \dot{\phi}^{2}$ plus some potential energy $V(\phi)$. If the slow-roll parameters, $\epsilon \equiv\left(M_{\mathrm{Pl}}^{2} / 16 \pi\right)\left(V^{\prime} / V\right)^{2}$ and $\eta \equiv\left(M_{\mathrm{Pl}}^{2} / 8 \pi\right)\left(V^{\prime \prime} / V\right)$, are small, then the field rolls slowly. The energy density is then dominated by the potential energy, the pressure is negative, and the expansion of the Universe is inflationary.

Quantum fluctuations in the inflaton give rise to primordial density perturbations characterized by a gravitationalpotential power spectrum $P_{\Phi}(k) \propto V / \epsilon$, where $V$ and $\epsilon$ are evaluated at the value the inflaton took when the comoving wave number $k$ exited the horizon during inflation. Differentiation of the expression for $P_{\Phi}(k)$ suggests that the power spectrum can be approximated as $P_{\Phi}(k) \propto$ $k^{n_{s}-1}$, where the scalar spectral index $n_{s}=1-6 \epsilon+2 \eta$ is close to unity, consistent with current measurements $[3,13]$.

The power spectrum $P_{\Phi}(k)$ may vary with $k$ because different values of $k$ sample the quantity $V / \epsilon$ at different values of the inflaton $\phi$. This suggests that the power asymmetry might be explained by a large-amplitude mode of $\phi$ with a comoving wavelength that is long compared with the current Hubble distance $\left(k \ll H_{0}\right)$. Then one side of the CMB sky would reflect the imprint of a different value of $\phi$ than the other side. From $P_{\Phi}(k) \propto$ $V / \epsilon$, we infer a fractional power asymmetry,

$$
A \equiv \frac{\Delta P_{\Phi}}{P_{\Phi}}=-2 \sqrt{\frac{\pi}{\epsilon}}\left(1-n_{s}\right) \frac{\Delta \phi}{M_{\mathrm{Pl}}},
$$

where $\Delta \phi$ is the change in the inflaton field across the observable Universe. A $10 \%$ variation in the amplitude of the CMB temperature fluctuations corresponds to a power asymmetry $A=0.2$.

The gravitational-potential perturbation $\Phi$ during matter domination is related to the inflaton perturbation $\delta \phi$ through $\Phi=(6 / 5) \sqrt{\pi / \epsilon}\left(\delta \phi / M_{\mathrm{Pl}}\right)$. Thus, a longwavelength perturbation $\delta \phi \propto \sin [\vec{k} \cdot \vec{x}+\varpi]$, with $k x_{\mathrm{d}} \ll 1$ (where $x_{\mathrm{d}}$ is the distance to the surface of last scatter), introduces a gravitational-potential perturbation with the same spatial dependence. It follows from Eq. (1) that $\Delta \Phi=3 A /\left[5\left(n_{s}-1\right)\right]$. An immediate concern, therefore, is whether this large-amplitude perturbation is consistent with the isotropy of the CMB.

Gravitational-potential perturbations give rise to temperature fluctuations in the CMB through the SachsWolfe effect [14] $(\delta T / T \simeq \Phi / 3)$. A large-scale potential perturbation might thus be expected to produce a $\mathrm{CMB}$ temperature dipole of similar magnitude. However, for the Einstein-de Sitter universe, the potential perturbation induces a peculiar velocity whose Doppler shift cancels the intrinsic temperature dipole [15]. The same is true for a flat universe with a cosmological constant [10].
Although the dipole vanishes, measurements of the CMB temperature quadrupole and octupole constrain the cosmological potential gradient $[15,16]$. Here we outline how these constraints are derived; the full calculation is presented elsewhere [10]. Since $k x_{\mathrm{d}} \ll 1$, we first expand the sinusoidal dependence $\Phi(\vec{x})=\Phi_{\vec{k}} \sin (\vec{k} \cdot \vec{x}+\varpi)$ in powers of $\vec{k} \cdot \vec{x}$. Then the terms that contribute to the CMB quadrupole and octupole are

$$
\Phi(\vec{x})=-\Phi_{\vec{k}}\left\{\frac{(\vec{k} \cdot \vec{x})^{2}}{2} \sin \varpi+\frac{(\vec{k} \cdot \vec{x})^{3}}{6} \cos \varpi\right\} .
$$

The CMB temperature anisotropy produced by the potential in Eq. (2) is

$$
\frac{\Delta T}{T}(\hat{n})=-\Phi_{\vec{k}}\left[\frac{\mu^{2}}{2}\left(k x_{\mathrm{d}}\right)^{2} \delta_{2} \sin \varpi+\frac{\mu^{3}}{6}\left(k x_{\mathrm{d}}\right)^{3} \delta_{3} \cos \varpi\right],
$$

where $\mu \equiv \hat{k} \cdot \hat{n}$ and $\Phi_{\vec{k}}$ is evaluated at the time of decoupling $\left(\tau_{\mathrm{d}}\right)$. The $\delta_{i}$ account for the Sachs-Wolfe (including integrated) effect and the Doppler effect induced by $\Phi_{\vec{k}}$; for a $\Lambda$ CDM universe with $\Omega_{M}=0.28$ and decoupling redshift $z_{\mathrm{d}}=1090$, we find that $\delta_{2}=0.33$ and $\delta_{3}=0.35$. Choosing $\hat{k}=\hat{z}$, Eq. (3) gives nonzero values for the spherical-harmonic coefficients $a_{20}$ and $a_{30}$. The relevant observational constraints are therefore

$$
\begin{aligned}
& \left(k x_{\mathrm{d}}\right)^{2}\left|\Phi_{\vec{k}}\left(\tau_{\mathrm{d}}\right) \sin \varpi\right| \lesssim 5.8 Q, \\
& \left(k x_{\mathrm{d}}\right)^{3}\left|\Phi_{\vec{k}}\left(\tau_{\mathrm{d}}\right) \cos \varpi\right| \lesssim 32 \mathcal{O},
\end{aligned}
$$

where $Q$ and $\mathcal{O}$ are upper bounds on $\left|a_{20}\right|$ and $\left|a_{30}\right|$, respectively, in a coordinate system aligned with the power asymmetry. We take $Q=3 \sqrt{C_{2}} \lesssim 1.8 \times 10^{-5}$ and $\mathcal{O}=$ $3 \sqrt{C_{3}} \lesssim 2.7 \times 10^{-5}, 3$ times the measured rms values of the quadrupole and octupole [17], as $3 \sigma$ upper limits; this accounts for cosmic variance in the quadrupole and octupole due to smaller-scale modes. The temperature quadrupole and octupole induced by the superhorizon mode can be made arbitrarily small for fixed $\Delta \Phi \simeq \Phi_{\vec{k}}\left(k x_{\mathrm{d}}\right) \cos \varpi$ by choosing $k$ to be sufficiently small. However, we also demand that $\Phi_{\vec{k}} \lesssim 1$ everywhere, and this sets a lower bound on $\left(k x_{\mathrm{d}}\right)$.

We now return to the power asymmetry generated by an inflaton perturbation. The largest value of $\Delta \Phi$ is obtained if $\varpi=0$, in which case the perturbation produces no quadrupole. The octupole constraint [Eq. (5)] combined with $\left(k x_{\mathrm{d}}\right) \gtrsim|\Delta \Phi|$ [i.e., the requirement $\Phi_{\vec{k}} \lesssim 1$ ] implies that $|\Delta \Phi| \lesssim(32 \mathcal{O})^{1 / 3}$. Given that $\left(1-n_{s}\right) \lesssim 0.06$, we see that the maximum possible power asymmetry obtainable with a single superhorizon mode is $A_{\max } \simeq 0.1(32 \mathcal{O})^{1 / 3} \simeq$ 0.0095 . This is too small, by more than an order of magnitude, to account for the observed asymmetry. The limit can be circumvented if a number of Fourier modes conspire to make the density gradient across the observable Universe 
smoother. This would require, however, that we live in a very special place in a very unusual density distribution.

\section{THE CURVATON MODEL}

We thus turn our attention to the curvaton model [11] of inflation. This model introduces a second scalar field $\sigma$, the curvaton, with potential $V(\sigma)=(1 / 2) m_{\sigma}^{2} \sigma^{2}$. During inflation, it is effectively massless, $m_{\sigma} \ll H_{I}$ (where $H_{I}$ is the inflationary expansion rate), and its density is negligible. Its homogeneous value $\bar{\sigma}$ remains classically frozen during inflation, but quantum effects give rise to fluctuations $\delta \sigma$ of rms amplitude $(\delta \sigma)_{\mathrm{rms}} \simeq\left(H_{I} / 2 \pi\right)$. Well after inflation, the curvaton rolls toward its minimum and then later oscillates about its minimum-i.e., a cold gas of $\sigma$ particles. These particles then decay to radiation. The fluctuations in the curvaton field will produce gravitationalpotential perturbations with a power spectrum,

$$
P_{\Phi, \sigma} \propto R^{2}\left\langle\left[\frac{\delta V}{V(\bar{\sigma})}\right]^{2}\right\rangle \sim R^{2}\left(\frac{H_{I}}{\pi \bar{\sigma}}\right)^{2},
$$

provided that $\bar{\sigma} \gg H_{I}$ [18]. Here $R \equiv\left(\rho_{\sigma} / \rho_{\text {tot }}\right)$ is the energy density of the curvaton field just prior to its decay divided by the total energy density of the Universe at that time.

We hypothesize that the density due to curvaton decay is small compared with the density due to inflaton decay; i.e., $R \ll 1$. In this case, the perturbation in the total energy density, and thus the potential perturbation $\Phi$, due to a fluctuation in $\rho_{\sigma}$ will be suppressed, making it possible to satisfy the homogeneity conditions set by the CMB [Eqs. (4) and (5)], even if $\rho_{\sigma}$ has order-unity variations. We then hypothesize that the power asymmetry comes from a variation $\Delta \bar{\sigma}$ in the value of the mean curvaton field across the observable Universe. Since $R \propto \bar{\sigma}^{2}$ for $R \ll 1$, the power spectrum for gravitational-potential perturbations produced by the curvaton is proportional to $\bar{\sigma}^{2}$. A variation $\Delta \bar{\sigma}$ in the value of the mean curvaton field across the observable Universe therefore induces a fractional power asymmetry $\Delta P_{\Phi, \sigma} / P_{\Phi, \sigma} \simeq 2(\Delta \bar{\sigma} / \bar{\sigma})$.

First we must ensure that this inhomogeneity does not violate Eqs. (4) and (5). The potential fluctuation during matter domination produced by a fluctuation $\delta \sigma$ in the curvaton field is

$$
\Phi=-\frac{R}{5}\left[2\left(\frac{\delta \sigma}{\bar{\sigma}}\right)+\left(\frac{\delta \sigma}{\bar{\sigma}}\right)^{2}\right] .
$$

Consider a superhorizon sinusoidal perturbation to the curvaton field $\delta \bar{\sigma}=\sigma_{k} \sin (\vec{k} \cdot \vec{x}+\varpi)$. If we ignored the term in Eq. (7) quadratic in $\delta \sigma$, then the upper bound to $\delta \bar{\sigma}$ would be obtained by setting $\varpi=0$. As with the inflaton, the constraint would then arise from the CMB octupole. However, the term in Eq. (7) quadratic in $\delta \sigma$ gives rise to a term in $\Phi$ quadratic in $(\vec{k} \cdot \vec{x})$-i.e.,
$\Phi_{\text {quad }}=-(R / 5)\left(\sigma_{k} / \bar{\sigma}\right)^{2}(\vec{k} \cdot \vec{x})^{2}$ for $\varpi=0$. Noting that $(\Delta \bar{\sigma} / \bar{\sigma})=\left(\sigma_{k} / \bar{\sigma}\right)\left(\vec{k} \cdot \vec{x}_{\mathrm{d}}\right)$, the quadrupole bound in Eq. (4) yields an upper limit,

$$
R\left(\frac{\Delta \bar{\sigma}}{\bar{\sigma}}\right)^{2} \lesssim \frac{5}{2}(5.8 Q)
$$

While this bound was derived for $\varpi=0$, most other values for $\varpi$ yield similar constraints [10].

Most generally, the primordial power will be some combination of that due to the inflaton and curvaton [19], $P_{\Phi}=P_{\Phi, \phi}+P_{\Phi, \sigma} \simeq 10^{-9}$, with a fraction $\xi \equiv P_{\Phi, \sigma} / P_{\Phi}$ due to the curvaton. The required asymmetry, $A \simeq$ $2 \xi(\Delta \bar{\sigma} / \bar{\sigma})$, can be obtained without violating Eq. (8) by choosing $R \leqq 58 Q \xi^{2} / A^{2}$, as shown in Fig. 2.

The only remaining issue is the Gaussianity of primordial perturbations. The curvaton fluctuation $\delta \sigma$ is a Gaussian random variable. Since the curvaton-induced density perturbation has a contribution quadratic in $\delta \sigma$, it implies a non-Gaussian contribution to the density fluctuation. The departure from Gaussianity can be estimated from the parameter $f_{\mathrm{NL}}$ [20], which for the curvaton model is $f_{\mathrm{NL}} \simeq 5 \xi^{2} /(4 R)$ [21-23]. The current upper limit, $f_{\mathrm{NL}} \lesssim$ 100 [24], leads to the lower limit to $R$ shown in Fig. 2.
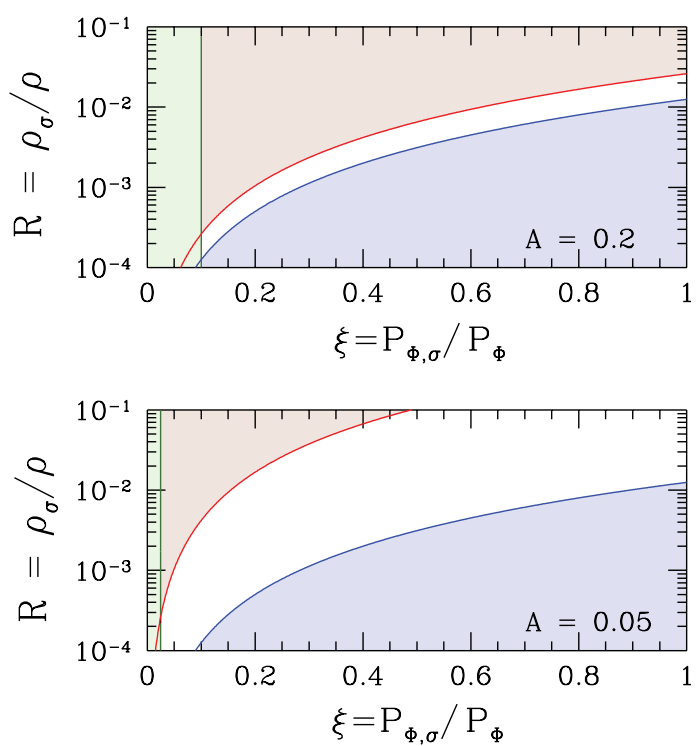

FIG. 2 (color online). The $R-\xi$ parameter space for the curvaton model that produces a power asymmetry $A=0.2$ (top panel) and $A=0.05$ (bottom panel). Here $R$ is the fraction of the cosmological density due to curvaton decay, and $\xi$ is the fraction of the power due to the curvaton. The upper limit to $R$ comes from the CMB-quadrupole constraint. The lower bound comes from $f_{\mathrm{NL}} \leq 100$. The lower limit to $\xi$ comes from the requirement that the fractional change in the curvaton field across the observable Universe be less than 1 . If $A$ is lowered, the lower bound to $R$ remains unchanged, but the upper bound increases, proportional to $A^{-2}$. The lower limit to $\xi$ also decreases as $A$ decreases, proportional to $A$. 
Figure 2 shows that there are values of $R$ and $\xi$ that lead to a power asymmetry $A=0.2$ and are consistent with measurements of the $\mathrm{CMB}$ quadrupole and $f_{\mathrm{NL}}$. For any value of $A$, the allowed region of $R-\xi$ parameter space is

$$
\frac{5}{4 f_{\mathrm{NL}, \max }} \lessgtr \frac{R}{\xi^{2}} \lessgtr 58 \frac{Q}{A^{2}},
$$

where $f_{\mathrm{NL}, \max }$ is the largest allowed value for $f_{\mathrm{NL}}$. Thus, we see that measurements of the $\mathrm{CMB}$ quadrupole and $f_{\mathrm{NL}}$ place an upper bound,

$$
A \lesssim \sqrt{(58 Q)\left(\frac{4 f_{\mathrm{NL}, \max }}{5}\right)},
$$

on the power asymmetry that may be generated by a superhorizon curvaton fluctuation. For $Q=1.8 \times 10^{-5}$, we predict (for $A \simeq 0.2$ ) $f_{\mathrm{NL}} \gtrsim 50$, much larger than $f_{\mathrm{NL}} \ll$ 1 predicted by standard slow-roll inflation. Values as small as $f_{\mathrm{NL}} \simeq 5$ should be accessible to the forthcoming Planck satellite, and so there should be a clear signature in Planck if the power asymmetry was generated by a curvaton perturbation and $A=0.2$.

If $(\delta \sigma / \bar{\sigma}) \ll 1$, the power due to the curvaton is $P_{\Phi, \sigma} \simeq$ $(2 R / 5)^{2}\left\langle(\delta \sigma / \bar{\sigma})^{2}\right\rangle$. The power required from the curvaton fixes $R(\delta \sigma / \bar{\sigma})_{\mathrm{rms}} \simeq 8 \times 10^{-5} \xi^{1 / 2}$, from which it follows that $(\delta \sigma / \bar{\sigma})_{\text {rms }} \lesssim 0.2$ for the allowed parameter space in Fig. 2, thus verifying that this parameter is small. We find from $(\Delta \bar{\sigma} / \bar{\sigma})=A / 2 \xi \lesssim 1$ that the required cross-horizon variation $\Delta \bar{\sigma} / \bar{\sigma}$ in the curvaton is large compared with the characteristic quantum-mechanical curvaton fluctuation $(\delta \sigma / \bar{\sigma})_{\mathrm{rms}}$; the required $\Delta \bar{\sigma}$ is at least a $\sim 5 \sigma$ fluctuation. It may therefore be that this large-scale mode is a superhorizon inhomogeneity not completely erased by inflation. Another possibility is that positive- and negative-value cells of $\bar{\sigma}$ created during inflation may be large enough to encompass the observable Universe; if so, we would observe an order-unity fluctuation in $\bar{\sigma}$ near the $\bar{\sigma}=0$ wall that divides two cells [12].

\section{SUMMARY AND DISCUSSION}

The hemispherical power asymmetry in the CMB challenges the assumption that the Universe is isotropic and homogeneous. A superhorizon perturbation in an inflationary field would introduce a preferred direction in the Universe, and we have investigated this mechanism for generating the observed power asymmetry. We found that the required superhorizon fluctuation in the inflaton field is inconsistent with measurements of the CMB octupole. A superhorizon fluctuation in a subdominant scalar field, however, is a viable alternative. A superhorizon curvaton perturbation can generate the observed power asymmetry without introducing unacceptable anisotropy and nonGaussianity in the CMB.

We have considered the specific asymmetry $A \simeq 0.2$ reported for WMAP, but our results can be scaled for different values of $A$, should the measured value for the asymmetry change in the future. In particular, the $f_{\mathrm{NL}}$ constraint (the lower bound to $R$ ) in Fig. 2 remains the same, but the upper bound (from the quadrupole) increases as $A$ is decreased. The lower limit to $\xi$ also decreases as $A$ is decreased. Here we have also considered a general model in which primordial perturbations come from some combination of the inflaton and curvaton. Although it may seem unnatural to expect the two field decays to produce comparable fluctuation amplitudes, our mechanism works even if $\xi=1$ (the fluctuations are due entirely to the curvaton). Thus, the coincidence is not a requirement of the model.

If the power asymmetry can indeed be attributed to a superhorizon curvaton mode, then the workings of inflation are more subtle than the simplest models would suggest. Fortunately, the theory makes a number of predictions that can be pursued with future experiments. To begin, the modulated power should produce signatures in the $\mathrm{CMB}$ polarization and temperature-polarization correlations [25]. The curvaton model predicts non-Gaussianity, of amplitude $f_{\mathrm{NL}} \gtrsim 50$ for $A \simeq 0.2$, which will soon be experimentally accessible. However, the theory also predicts that the small-scale non-Gaussianity will be modulated across the sky by the variation in $\bar{\sigma}$ (and thus in $\xi$ and $R$ ). The presence of curvaton fluctuations also changes other features of the CMB [23]. The ratio of tensor and scalar perturbations $(r)$ is reduced by a factor of $(1-\xi)$ and the scalar spectral index is $n_{s}=1-2 \epsilon-(1-\xi) \times$ $(4 \epsilon-2 \eta)$. The tensor spectral index $\left(n_{T}\right)$, however, is unaltered by the presence of the curvaton, and so this model alters the inflationary consistency relation between $n_{T}$ and $r$ and possibly the prospects for testing it [26].

Here we have assumed simply that the curvaton decays to the same mixture of baryons, dark matter, and radiation as the inflaton. However, if the inflaton and curvaton decay products differ, then there may be an isocurvature component $[21,27]$. Finally, the simplest scenario predicts a scaleinvariant power asymmetry; the asymmetry has been found at multipole moments $\ell \lesssim 40$, but there are claims that it does not extend to higher $\ell$ [28]. If this result holds, it will be interesting to see whether the departure from scale invariance can be obtained by suitably altering the power spectra for the curvaton and inflaton. For instance, a sudden drop in both $V^{\prime}(\phi)$ and $V(\phi)$ could enhance the gravitational-potential fluctuations from the inflaton while suppressing the fluctuations from the curvaton [7]; the resulting drop in $\xi$ would reduce the power asymmetry on smaller scales. We leave such elaborations for future work.

\section{ACKNOWLEDGMENTS}

We thank K. Górski and H. K. Eriksen for discussions. This work was supported by DOE Grant No. DE-FG03-92ER40701 and the Gordon and Betty Moore Foundation. 
[1] A. H. Guth, Phys. Rev. D 23, 347 (1981); A. Albrecht and P. J. Steinhardt, Phys. Rev. Lett. 48, 1220 (1982); A. D. Linde, Phys. Lett. 108B, 389 (1982).

[2] P. de Bernardis et al. (Boomerang Collaboration), Nature (London) 404, 955 (2000).

[3] J. Dunkley et al. (WMAP Collaboration), arXiv:0803.0586.

[4] S. Cole et al. (2dFGRS Collaboration), Mon. Not. R. Astron. Soc. 362, 505 (2005); S. Dodelson et al. (SDSS Collaboration), Astrophys. J. 572, 140 (2002).

[5] H. K. Eriksen et al., Astrophys. J. 605, 14 (2004); 609, 1198(E) (2004); F. K. Hansen, A. J. Banday, and K. M. Górski, Mon. Not. R. Astron. Soc. 354, 641 (2004); H. K. Eriksen et al., Astrophys. J. 660, L81 (2007).

[6] A. de Oliveira-Costa et al., Phys. Rev. D 69, 063516 (2004); K. Land and J. Magueijo, Phys. Rev. Lett. 95, 071301 (2005).

[7] C. Gordon, Astrophys. J. 656, 636 (2007).

[8] J.F. Donoghue, K. Dutta, and A. Ross, arXiv:astro-ph/ 0703455.

[9] C. Gordon, W. Hu, D. Huterer, and T. M. Crawford, Phys. Rev. D 72, 103002 (2005).

[10] A. L. Erickcek, S. M. Carroll, and M. Kamionkowski, Phys. Rev. D 78, 083012 (2008).

[11] S. Mollerach, Phys. Rev. D 42, 313 (1990); A. D. Linde and V. F. Mukhanov, Phys. Rev. D 56, R535 (1997); D. H. Lyth and D. Wands, Phys. Lett. B 524, 5 (2002); T. Moroi and T. Takahashi, Phys. Lett. B 522, 215 (2001); 539, 303 (E) (2002).

[12] A. D. Linde and V.F. Mukhanov, J. Cosmol. Astropart. Phys. 04 (2006) 009.

[13] L. Verde and H. Peiris, J. Cosmol. Astropart. Phys. 07 (2008) 009.

[14] R. K. Sachs and A.M. Wolfe, Astrophys. J. 147, 73 (1967).

[15] L. P. Grishchuk and Ia. B. Zel'dovich, Astron. Zh. 55, 209
(1978) [Sov. Astron. 22, 125 (1978)]; M. S. Turner, Phys. Rev. D 44, 3737 (1991).

[16] P. G. Castro, M. Douspis, and P. G. Ferreira, Phys. Rev. D 68, 127301 (2003).

[17] G. Efstathiou, Mon. Not. R. Astron. Soc. 348, 885 (2004).

[18] K. A. Malik, D. Wands, and C. Ungarelli, Phys. Rev. D 67, 063516 (2003).

[19] D. Langlois and F. Vernizzi, Phys. Rev. D 70, 063522 (2004).

[20] L. Verde et al., Mon. Not. R. Astron. Soc. 313, L141 (2000); E. Komatsu and D. N. Spergel, Phys. Rev. D 63, 063002 (2001).

[21] D. H. Lyth, C. Ungarelli, and D. Wands, Phys. Rev. D 67, 023503 (2003).

[22] K. A. Malik and D. H. Lyth, J. Cosmol. Astropart. Phys. 09 (2006) 008.

[23] K. Ichikawa, T. Suyama, T. Takahashi, and M. Yamaguchi, Phys. Rev. D 78, 023513 (2008).

[24] E. Komatsu et al. (WMAP Collaboration), Astrophys. J. Suppl. Ser. 148, 119 (2003); A. P. S. Yadav and B. D. Wandelt, Phys. Rev. Lett. 100, 181301 (2008); E. Komatsu et al. (WMAP Collaboration), arXiv:0803.0547; A. Slosar, C. Hirata, U. Seljak, S. Ho, and N. Padmanabhan, J. Cosmol. Astropart. Phys. 08 (2008) 031.

[25] A. R. Pullen and M. Kamionkowski, Phys. Rev. D 76, 103529 (2007); C. Dvorkin, H. V. Peiris, and W. Hu, Phys. Rev. D 77, 063008 (2008).

[26] T. L. Smith, H. V. Peiris, and A. Cooray, Phys. Rev. D 73, 123503 (2006).

[27] M. Lemoine and J. Martin, Phys. Rev. D 75, 063504 (2007).

[28] E. P. Donoghue and J.F. Donoghue, Phys. Rev. D 71, 043002 (2005); B. Lew, J. Cosmol. Astropart. Phys. 08 (2008) 017. 